EPJ Web of Conferences 19, 06009 (2012)

DOI: $10.1051 /$ epjconf/20121906009

(C) Owned by the authors, published by EDP Sciences, 2012

\title{
Elemental abundances in AGB stars and the formation of the Galactic bulge
}

\author{
S. Uttenthaler 1 ,a , J.A.D.L. Blommaert ${ }^{2}$, T. Lebzelter ${ }^{1}$, N. Ryde ${ }^{3}$, P.R. Wood ${ }^{4}$, \\ M. Schultheis ${ }^{5}$ and B. Aringer ${ }^{6}$
}

1 University of Vienna, Department of Astronomy, Türkenschanzstraße 17, 1180 Vienna, Austria

2 Instituut voor Sterrenkunde, K. U. Leuven, Celestijnenlaan 200D, 3000 Leuven, Belgium

3 Department of Astronomy and Theoretical Physics, Box 43, 22100 Lund, Sweden

4 Research School of Astronomy and Astrophysics, Australian National University, Cotter Rd, Weston Creek ACT 2611, Australia

5 Observatoire de Besançon, 41bis, avenue de l'Observatoire, 25000 Besançon, France

6 INAF - Padova Astronomical Observatory, Vicolo dell'Osservatorio 5, 35122 Padova, Italy

\begin{abstract}
We obtained high-resolution near-IR spectra of 45 AGB stars located in the Galactic bulge. The aim of the project is to determine key elemental abundances in these stars to help constrain the formation history of the bulge. A further aim is to link the photospheric abundances to the dust species found in the winds of the stars. Here we present a progress report of the analysis of the spectra.
\end{abstract}

\section{INTRODUCTION}

The bulge of the Milky Way Galaxy is one of its major components, but its formation is still poorly understood. It still holds many surprises for us, see e.g. the recently discovered double red clump [1, 2]. Elemental abundances in bulge stars are an important tool to constrain formation scenarios. K/M giants and $\mathrm{PNe}$ in the bulge have already been studied for their abundance patterns, but not the evolutionary state between them, the AGB. We obtained high-resolution near-IR spectra of 45 AGB stars with the CRIRES@VLT spectrograph to better characterise the giant star population in the bulge.

\section{THE SAMPLE}

The CRIRES targets are located at various Galactic latitudes, from $b=-1^{\circ}$ to $b=-12^{\circ}$, but most of them are less than $4^{\circ}$ south of the Galactic plane. The stars have been selected either from ISOGAL photometry to cover a range in $K-$ [15] (i.e. mass-loss rate), or from the sample of outer bulge AGB stars that has been observed with UVES by [3]. There are several indications that suggest an intermediate age of bulge AGB variables, e.g. their pulsation period distribution [4] and the presence of technetium in some of them [3]. For these reasons, it is interesting to compare their chemical abundances to other populations in the bulge.

\footnotetext{
a e-mail: stefan.uttenthaler@univie.ac.at
}

This is an Open Access article distributed under the terms of the Creative Commons Attribution-Noncommercial License 3.0, which permits unrestricted use, distribution, and reproduction in any noncommercial medium, provided the original work is properly cited. 


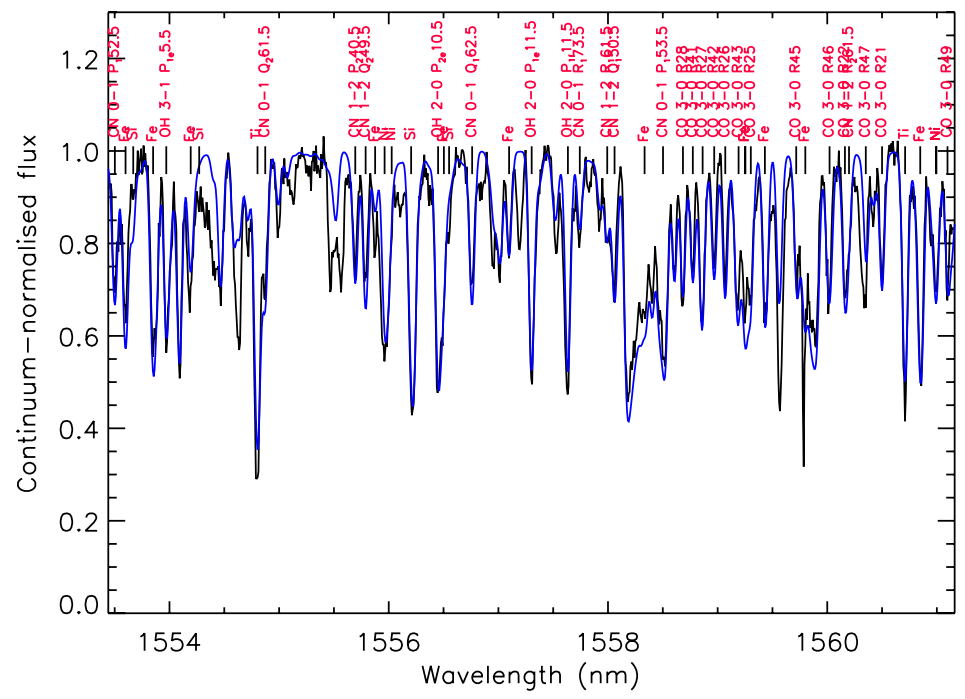

Figure 1. Spectrum of the star J174924.1-293522 on chip 2 of the H-band CRIRES setting (black). The blue graph is a synthetic spectrum based on COMARCS models with $T_{\text {eff }}=3700 \mathrm{~K}$ and $\log g=+0.30$. It was interpolated within the 80 grid models to the best-fit overall abundance pattern of $[\mathrm{Fe} / \mathrm{H}]=+0.15,[\alpha / \mathrm{Fe}]=-0.36$, and $\mathrm{C} / \mathrm{O}=0.48$

\section{ANALYSIS}

The target stars were mainly observed in one setting in the H-band and one setting in the K-band. These regions contain numerous atomic $(\mathrm{Fe}, \mathrm{Ti}, \mathrm{Si}, \ldots)$ and molecular lines $(\mathrm{OH}, \mathrm{CN}$, and $\mathrm{CO}$, including ${ }^{13} \mathrm{CO}$ ). They allow for the measurement of abundances of all the involved elemental species.

We employ hydrostatic COMARCS model atmospheres and COMA spectral synthesis [5] in the analysis of the spectra. Stellar parameters (luminosity, mass, temperature, $\log g$ ) have been estimated from photometry, low-resolution optical spectra, and the pulsation periods of the stars. For every star, a grid of model atmospheres and spectra with 80 different abundance patterns is calculated. We interpolate between the grid spectra to determine the best-fitting overall abundance pattern. A $\chi^{2}$ minimisation method is employed in this step. Of particular interest is the run of $[\alpha / \mathrm{Fe}]$ vs. $[\mathrm{Fe} / \mathrm{H}]$ because it will give information about the formation time scale of the population from which the AGB stars descend. Figure 1 shows an example of an observed spectrum, together with the best-fit interpolated spectrum.

\section{THE CONNECTION TO DUST}

We also have Spitzer/IRS spectra of all targets, from which their dominant dust species are known. A variety of dust feature is found, including amorphous silicates and aluminum oxide, but also the 13 and $19.5 \mu \mathrm{m}$ features with uncertain carriers [6]. One central aim of the project is to investigate the connection between photospheric abundances of key elements in the dust formation (e.g. $\mathrm{Mg}, \mathrm{Al}$ ) and the dust species found in the stellar wind. With our data we will be able to better understand the bulge, but also the AGB stars themselves ${ }^{1}$ !

1 This research was funded by the Austrian Science Fund (FWF): P22911-N16, P21988-N16, and P23737-N16. 
Assembling the Puzzle of the Milky Way

\section{References}

[1] Nataf D., et al., 2010, ApJL, 721, 28

[2] McWilliam A. \& Zoccali M., 2010, ApJ, 724, 1491

[3] Uttenthaler S., Hron J., Lebzelter T., et al., 2007, A\&A, 463, 251

[4] Groenewegen M. A. T. \& Blommaert J. A. D. L. 2005, A\&A, 443, 143

[5] Aringer B., Girardi L., Nowotny W., et al., 2009, A\&A, 503, 913

[6] Vanhollebeke E., 2007, Ph.D. thesis, Katholieke Universiteit Leuven, Belgium 